\title{
Prevención en salud mental, familia, escuela y sociedad
}

\author{
Raymundo Macías Avilés \\ Psiquiatra, Terapeuta Familiar y de Pareja, Psicoanalista. Presidente del Consejo Consultivo, Profesor y Supervisor del Instituto de la Familia, \\ A.C., Ex Presidente y Coordinador de la Sección Académica Psiquiatría y Espiritualidad, Asociación Psiquiátrica Mexicana, A.C.
}

\begin{abstract}
RESUMEN
Se plantea cómo las necesidades humanas, derivadas de las distintas dimensiones de nuestra naturaleza, se satisfacen adecuadamente o no por las funciones psicosociales de la familia, con valores relacionales positivos, a través del ciclo vital y el proceso de desarrollo dentro del contexto de interacción de los sistemas humanos. De lograrse esto, se podrá generar bienestar y salud mental; de lo contrario, podrán generarse problemas, conflictos y malestar, que tienden a mantenerse por circuitos recursivos de retroalimentación negativa, queja, contraqueja, acusación, contraacusación. Éstos con frecuencia nos atrapan en el pasado, en el resentimiento, en el rencor, en el "no cambio", pues lo que ya pasó es imposible cambiarlo, hacer que no hubiera pasado. Así mismo, se plantean distintos niveles de intervención, idealmente enfatizando actividades educativas y de promoción de salud mental, así como preventivas de algunas problemáticas frecuentes, antes de tener que intervenir frente a conflictos mayores, a veces muy difíciles, pero no imposibles de manejar.
\end{abstract}

Palabras clave: Naturaleza humana, interacción sistémica, familia, funciones psicosociales, valores relacionales, ciclo vital, problema y conflicto, niveles de intervención.

\begin{abstract}
It is proposed how human needs, derived from the different dimensions of our human nature, are adequately satisfied or not, by the family psycho-social functions, with positive relational values, through the life cycle and developmental process, within the context of our human systems interaction. If this is achieved, well-being feelings and mental health could be generated; otherwise problems, conflicts and ill-being feelings would occur and there might be a tendency to be manteined by recursive negative feedback circuits: complaint-countercomplaint, accusation-counteraccusation, these negative feedback circuits trap us in the past quite frequently, in resentment and rancor, in the position of "No change", because whatever has happened is impossible to make that didn't happened. Thus different levels of intervention are proposed, ideally emphasizing educational activities, as well as promotional mental heal th activities and preventive measures in face of the most common problems, before having to intervene facing greater conflicts, sometimes very difficult but not impossible to manage.
\end{abstract}

Keywords: Human nature, systems interaction, family, psychosocial functions, relational values, vital cycle, problem and conflict, intervention levels.

\section{Naturaleza humana}

Para hablar de la familia en la especie humana tenemos que hablar de la naturaleza del hombre y consideramos que son cuatro las dimensiones fundamentales que la constituyen: la biológica, la psicológica, la social y la espiritual, la dimensión psicológica considera a su vez lo conductual, lo conceptual y lo emocional (Figuras 1 y 2).

Por mucho tiempo se tomaron en cuenta solamente los tres primeros, por el dominio del positivismo prevalente en la ciencia, desde fines del siglo XIX y casi todo el XX, pero a partir del campo de la psicoterapia, en los últimos años se ha reconocido y recuperado la importancia de lo espiritual y el enorme valor que el sistema de creencias y valores tiene, para la mejor comprensión, formación y ayuda más integral de los seres humanos, tanto a nivel individual como familiar.

Es en la totalidad de sus dimensiones que tenemos que entenderlas y promover su desarrollo y realización más plena.

\section{NECESIDADES HUMANAS}

Derivadas de su naturaleza humana, cada ser humano tiene una serie de necesidades que podemos considerar divididas en dos grandes grupos, uno sería el de las Necesidades Biológicas de Subsistencia, tanto a nivel individual como de la especie y de las que se derivan los impulsos y tendencias instintivas tanto 
de conservación, como sexuales, integrando un gran instinto de vida.

El otro gran grupo es el de las necesidades más específicamente humanas, denominado así porque creemos que no son exclusivas o privativas del hombre, aunque en él alcanzan tal vez un mayor desarrollo. A su vez, y también a diferencia de Fromm, consideramos que no son solamente cinco necesidades, sino cinco grandes conjuntos de ellas, a cada uno de los que les damos un nombre compuesto, precisamente para denotar que no se trata de una sola necesidad, sino que comprende muchas otras más, bajo esa denominación binomial, que pretende ser autoexplicativa.

Finalmente cabe señalar que presentamos estas necesidades en un orden evolutivo, partimos desde las más básicas o fundamentales hasta llegar a las más desarrolladas o evolucionadas, y en este sentido se va ligando cada uno de los conjuntos con el inmediato, anterior y posterior.

\section{GRUPOS Y CONJUNTOS DE NECESIDADES HUMANAS}

- Necesidades biológicas de subsistencia, tanto a nivel individual como de la especie.

- Necesidades más específicamente humanas.

- Necesidades de relación-vinculación.

- Necesidades de aceptación-pertenencia.

- Necesidades de identidad-individualidad.

- Necesidades de marco de referencia-sistema de creencias y de valores.

- Necesidades de significado-trascendencia.

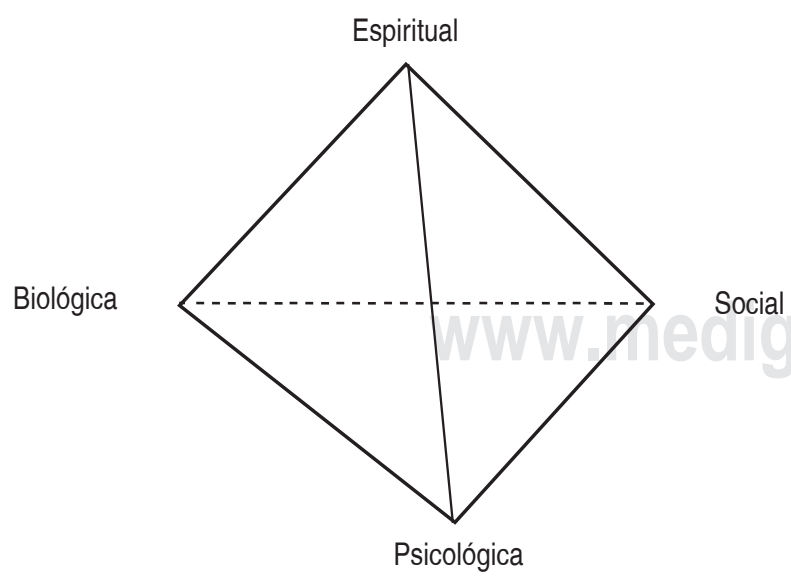

Figura 1: Naturaleza humana.

R. Macías-A.
Lo psicológico

Comportamiento

Lo conativo

Lo conductual

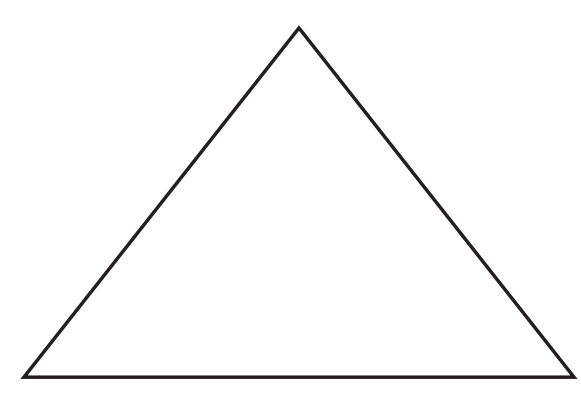

Entendimiento

Sentimiento

Lo cognitivo

Lo afectivo

Lo conceptual

Lo emocional

R. Macías-A.

Figura 2: Dimensión psicológica.

\section{INTERACCIÓN SISTÉMICA}

El Universo está organizado sistémicamente, es decir en sistemas, los que son conjuntos de elementos que interaccionan entre sí, de tal modo que el cambio de uno afecta al conjunto y viceversa, y que cumplen dos funciones de la morfostasis para mantener la estabilidad del sistema y la de morfogénesis para modificarse adaptativamente ante cambios de los sistemas de mayor amplitud que lo incluyen.

En los sistemas humanos la unidad sistémica más pequeña es el ser humano individual, podríamos decir el microsistema, que a su vez está integrado por subsistemas orgánicos-biológicos, como sistema nervioso central, vegetativo, sistema endocrino, sistema inmunológico, etcétera o psicológicos: el aparato intrapsíquico, el sistema de valores y creencias personales.

En un nivel sistémico superior encontramos a la pareja y la familia, mesosistemas o sistemas intermedios, puente entre el microsistema individual y el que le sigue a nivel macrosistema: el grupo social, étnico, religioso o finalmente en el nivel más alto la sociedad o la humanidad enteras. Estos sistemas interactúan entre sí y se influyen y retroinfluyen uno a otro, en ambos sentidos, incluso con saltos de nivel (Figura 3). 


\section{CONCEPTO Y TIPOS DE FAMILIAS}

Es preciso distinguir entre un concepto básico de familia, que se dará a continuación, como grupo primario de pertenencia, con raíces biológicas indispensables para la subsistencia de los individuos recién nacidos dentro de ella, y su desarrollo temprano, y que según antropólogos de la talla de Linton y Levi-Strauss, existe y existirá en tanto que el hombre se encuentre sobre la faz de la tierra, de las distintas formas, tipos o modelos de familias, que pueden cambiar e incluso aparecer o desaparecer a través del tiempo, en distintas culturas, épocas o latitudes.

Así podemos hablar de familia extensa, semiextensa, nuclear conyugal, uniparental, separada, reconstruida, extendida, binuclear, matri- o patriarcal, matri- o patrilineal, patri- o matrinominal, etcétera, pero sea cual sea su estructura o modalidad, obedece a una necesidad organizativa sistémica de integración grupal para poder responder a y satisfacer las necesidades humanas de sus miembros, en particular las más básicas de los más desvalidos.

En ese sentido, la familia es unidad sistémica de desarrollo y satisfacción de necesidades, funcional o disfuncional, generadora de salud y de bienestar o de malestar y problemática mental y emocional, pero asimismo, es unidad de estudio y de intervención educativa, promocional, preventiva o de tratamiento.

\section{CONCEPTO de FAMILIA}

Es un grupo de convivencia, de adscripción natural y/o pertenencia primaria, con o sin lazos de consanguinidad, en el que se comparte la escasez o abundancia de recursos de subsistencia y servicios, generalmente bajo el mismo techo, que constituye la unidad básica de producción y consumo de bienes, que incluye dos o más generaciones, al menos en alguna etapa de su ciclo vital, y que permite la subsistencia de sus miembros y facilita o no el desarrollo de sus potencialidades.

\section{FUNCIONES PSICOSOCIALES DE LA FAMILIA}

Las funciones psicosociales de la familia, señaladas en el siguiente cuadro, responden a las necesidades humanas que ya hemos visto.

Es preciso señalar además que no son privativas de la familia, como grupo de convivencia, cualquiera que fuese su modalidad, sino que pueden ser complementadas por otras instancias sociales, como la familia

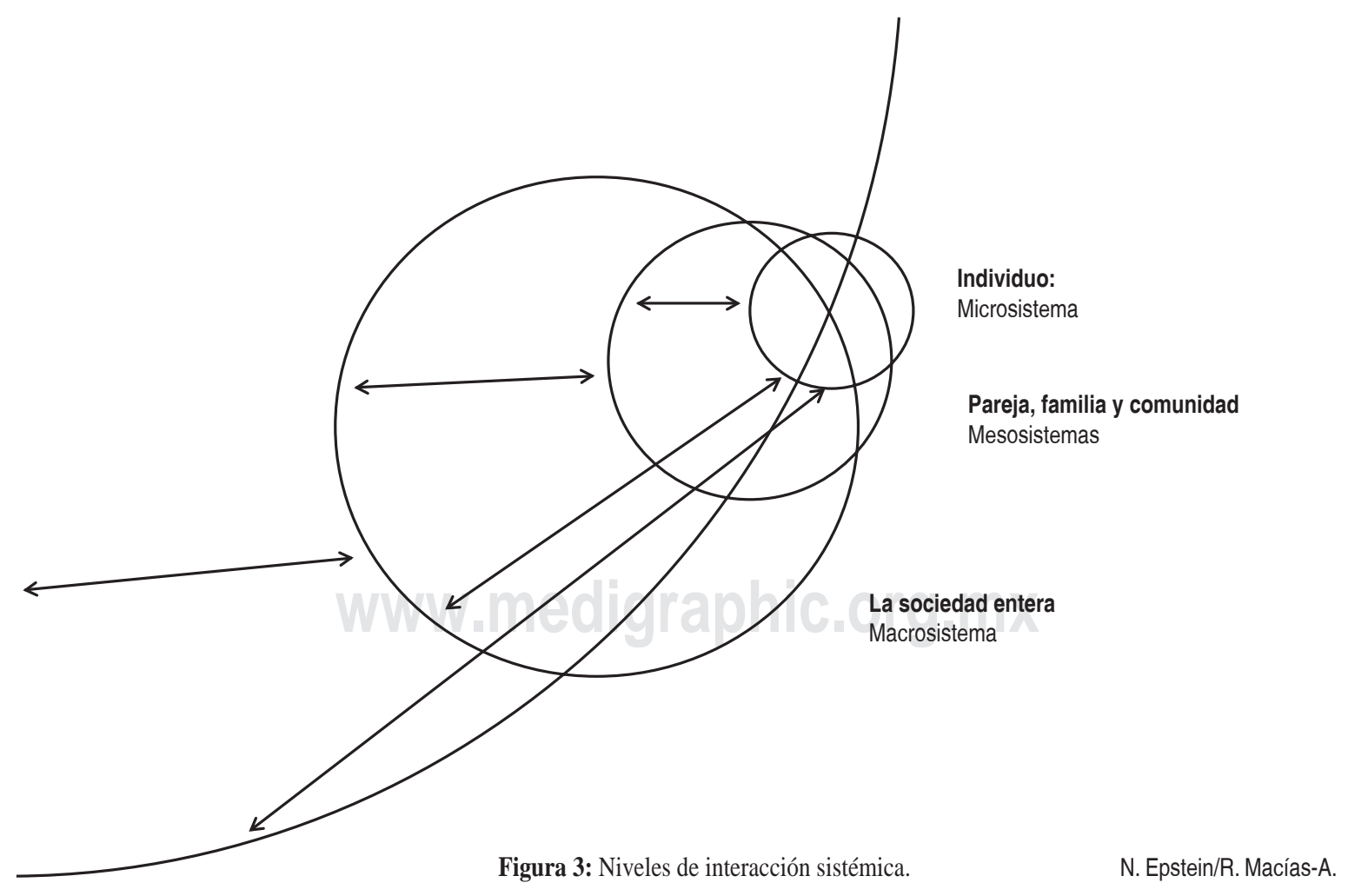




\begin{tabular}{|c|c|}
\hline \multicolumn{2}{|c|}{$\begin{array}{c}\text { Tabla 1: Funciones de } \\
\text { maternaje y de paternaje. }\end{array}$} \\
\hline Buen maternaje & Buen paternaje \\
\hline Vinculación & Provisión \\
\hline $\begin{array}{l}\text { Nutrición y protección, } \\
\text { aceptación incondicional, } \\
\text { confianza básica, autoestima, } \\
\text { seguridad afectiva }\end{array}$ & $\begin{array}{l}\text { Aceptación condicional, } \\
\text { estímulo, orientación, nor- } \\
\text { matividad, orden, responsa- } \\
\text { bilidad, trabajo }\end{array}$ \\
\hline \multicolumn{2}{|c|}{ Complementarias, sintónicas, no privativas } \\
\hline
\end{tabular}

R. Macías-A. extendida, los centros de desarrollo infantil, las escuelas, clubs sociales, funciones psicosociales de familia $y / 0$ deportivos, otros grupos de apoyo, etcétera; idealmente los medios podrían también contribuir a ello.

Del mismo modo, aunque algunas funciones fuesen más connaturales para ser realizadas por la madre, dada su condición biopsicosocial, las reconocemos como funciones de maternaje, en tanto que otro grupo de ellas, por la misma razón serían denominadas funciones de paternaje, no del padre. No son privativas de ninguno de ellos y se pueden e incluso deben complementarse en forma recíproca, integrativa y colaborativa por ambos, o bien por algún otro miembro del sistema familiar o por figuras sustitutas ante la ausencia de alguno de ellos (Tabla 1).
Funciones

Maternaje

Tendencia a desviación del desarrollo
Tendencia a desarrollo productivo

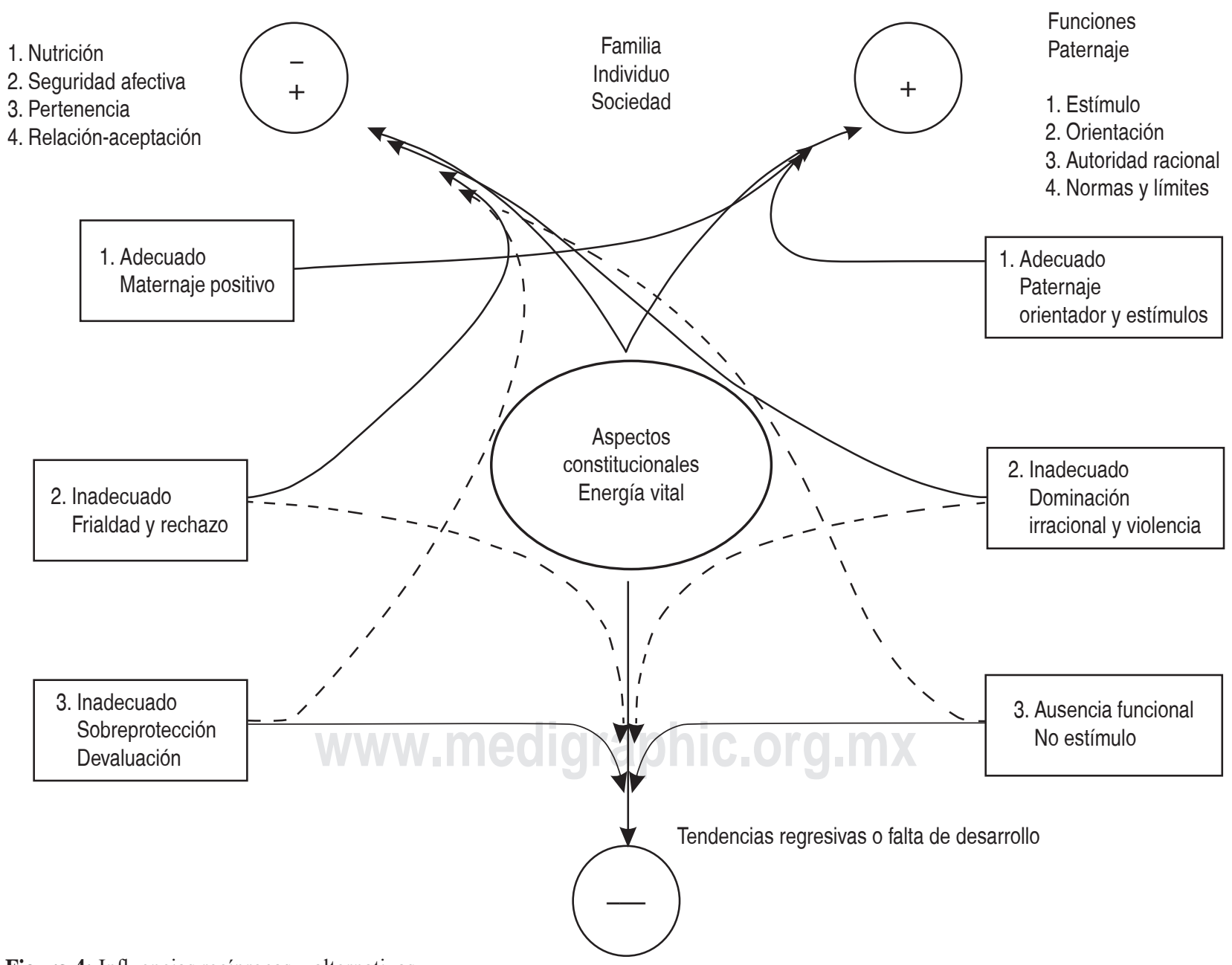

Figura 4: Influencias recíprocas y alternativas. 
Tabla 2: Las ocho crisis de las etapas

del desarrollo según Erik Erikson.

\begin{tabular}{lccc}
\hline Positiva & & Negativa & Valor \\
\hline Confianza & Nacimiento-año $1 / 2$ & Desconfianza & Esperanza \\
Autonomía & $1-3$ años & Vergüenza & Voluntad \\
Iniciativa & $4-5$ años & Culpa & Propósito \\
Industria & $6-11$ años & Inferioridad & Competencia \\
Identidad del yo & $12-20$ años & Confusión & Fidelidad \\
Intimidad & Juventud & Aislamiento & Amor \\
Generatividad & Madurez & Estancamiento & Cuidado \\
Integridad & Edad avanzada & Desesperanza & Sabiduría \\
\hline
\end{tabular}

Si estas funciones se cumplen satisfactoriamente, se puede generar un clima predominante de bienestar y armonía familiar, de no ser así nos podemos encontrar una familia disfuncional en una situación de malestar, disarmonía y conflictiva relacional de mayor o menor grado (Figura 4).

\section{Funciones psicosociales de familia}

1. Provee satisfacción a las necesidades biológicas de subsistencia.

2. Constituye la matriz emocional de las relaciones afectivas interpersonales.

3. Facilita el desarrollo de la identidad individual, ligada a la identidad familiar.

Tabla 3: Valores relacionales

(RICCAAS)

\begin{tabular}{lll}
\hline Reciprocidad & Respeto & Responsabilidad \\
Interés & Intimidad & Integridad \\
Comunicación & Comprensión & Congruencia \\
Compromiso & Compasión & Confianza \\
Aceptación & Adaptación & Apertura \\
Aprecio & Amor & Autonomía \\
Seguridad & Sinceridad & Solidaridad \\
\hline
\end{tabular}

R. Macías-A.
4. Provee los primeros modelos de identificación psicosexual.

5. Inicia el entrenamiento de los roles y organización sociales.

6. Educa, estimulando o no el aprendizaje y la creatividad.

7. Transmite valores, ideología y cultura (Tabla 1).

\section{Proceso de Desarkollo y Valores RELACIONALES}

El proceso de maduración y desarrollo del ser humano implica a su vez dos procesos, que aunque aparentan ser contrapuestos, son complementarios e integrativos: el de socialización, que está ligado a los primeros conjuntos de necesidades más específicamente humanas y el proceso de individuación, ligado a los conjuntos posteriores. La socialización tiene como valor primordial la solidaridad, el de la individuación es la autonomía (Figura 5).

Entendemos por valor relacional un principio normativo de conducta que conlleva como fin u objetivo plantear una forma de relacionarse más sana, que implicaría una disposición o actitud afectiva positiva y que se manifieste en una conducta concreta (Tabla 2).

\section{Ciclo Vital de la Familia y Valores RELACIONALES}

Se presentan las ocho edades del desarrollo del hombre según Erik Erikson, detallando en cada una la crisis 
Valor solidaridad

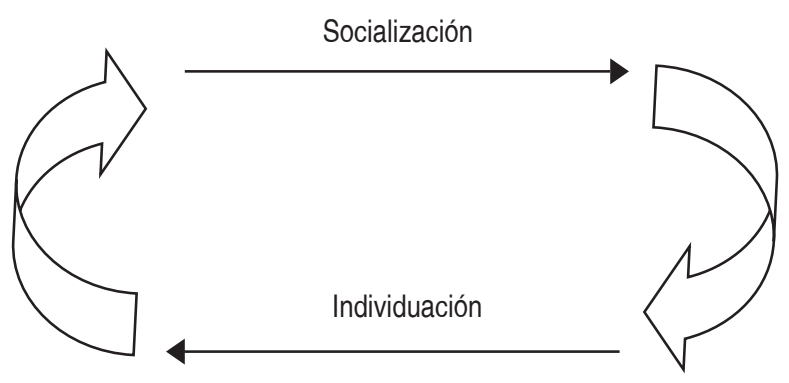

Valor autonomía

E. Fromm/R. Macías-A.

Figura 5: Proceso dialéctico de maduración y desarrollo.

entre el valor positivo a alcanzar, el negativo resultante de no lograr superar dicha crisis y el valor de logro cuando sí se puede (Tabla 3).

Se hace una propuesta de Ciclo Vital de la Familia, dividido en cuatro grandes etapas: los antecedentes, el inicio, el desarrollo y la declinación, divididas a su vez en fases, 14 en total (Tabla 4). En él se plantean, más de acuerdo a la idiosincrasia de las familias mexicanas y en forma desiderativa, las tareas y la forma que convendría cumplirlas para lograr un desarrollo más pleno y satisfactorio, tanto de los individuos como del sistema familiar.

Destacamos aquellas etapas y fases particularmente importantes para la educación y formación en los valores relacionales que se han mencionado.

\section{Proyecto De VIDA}

\section{Personal e interpersonal}

\section{Áreas}

1. Ocupacional y de realización personal.

2. Relaciones familiares y sociales.

3. Recreativa y de tiempo libre.

4. Afectivo-íntima.

5. Espiritual y/o de significados trascendentes.

\section{CONTRATOS RELACIONALES}

Se refieren al concepto de compromiso personal que uno está dispuesto a asumir y espera que asuma el otro, sobre el conjunto de beneficios-derechos y responsabi-
Tabla 4: Ciclo vital de pareja y familia.

\section{Los antecedentes \\ II. El inicio}

III. El desarrollo

(en ella ocurre la formación del sistema de creencias y valores, base de un posible proyecto de vida)

IV. La declinación
1. El desprendimiento de la familia de origen

2. El encuentro y el cortejo

3. El proyecto de pareja y la decisión de serlo

4. El matrimonio: compromiso de vida y constitución formal de la pareja

5. Integración inicial y decisión de ser familia

6. Advenimiento de los hijos. Nuevos roles

7. Familia e hijos pequeños. Lactancia, primera infancia

8. Familia y época preescolar

9. Los hijos y la escuela

10. Pubertad y adolescencia de los hijos

11. Los hijos jóvenes-adultos

12. El desprendimiento de los hijos, el nido vacío y el reencuentro

13. Las nuevas familias. Nuevos roles

14. Vejez y muerte, disolución de la familia nuclear
Según R. Macías-A.

lidades-obligaciones, conscientes o preconscientes, que uno espera tener en una relación interpersonal con un otro significativo. Incluye varios tipos de elementos basados en las necesidades más específicamente humanas, psicológicas y relacionales, algunos de esos elementos son derivados de expectativas no satisfechas adecuadamente en el pasado, que se depositan en la relación interpersonal presente y pueden ser foco de conflicto.

\section{Niveles de expectativas del contrato interpersonal}

1. Explícito, verbalizadas, conscientes.

2. Implícito, no verbalizadas, preconscientes. 


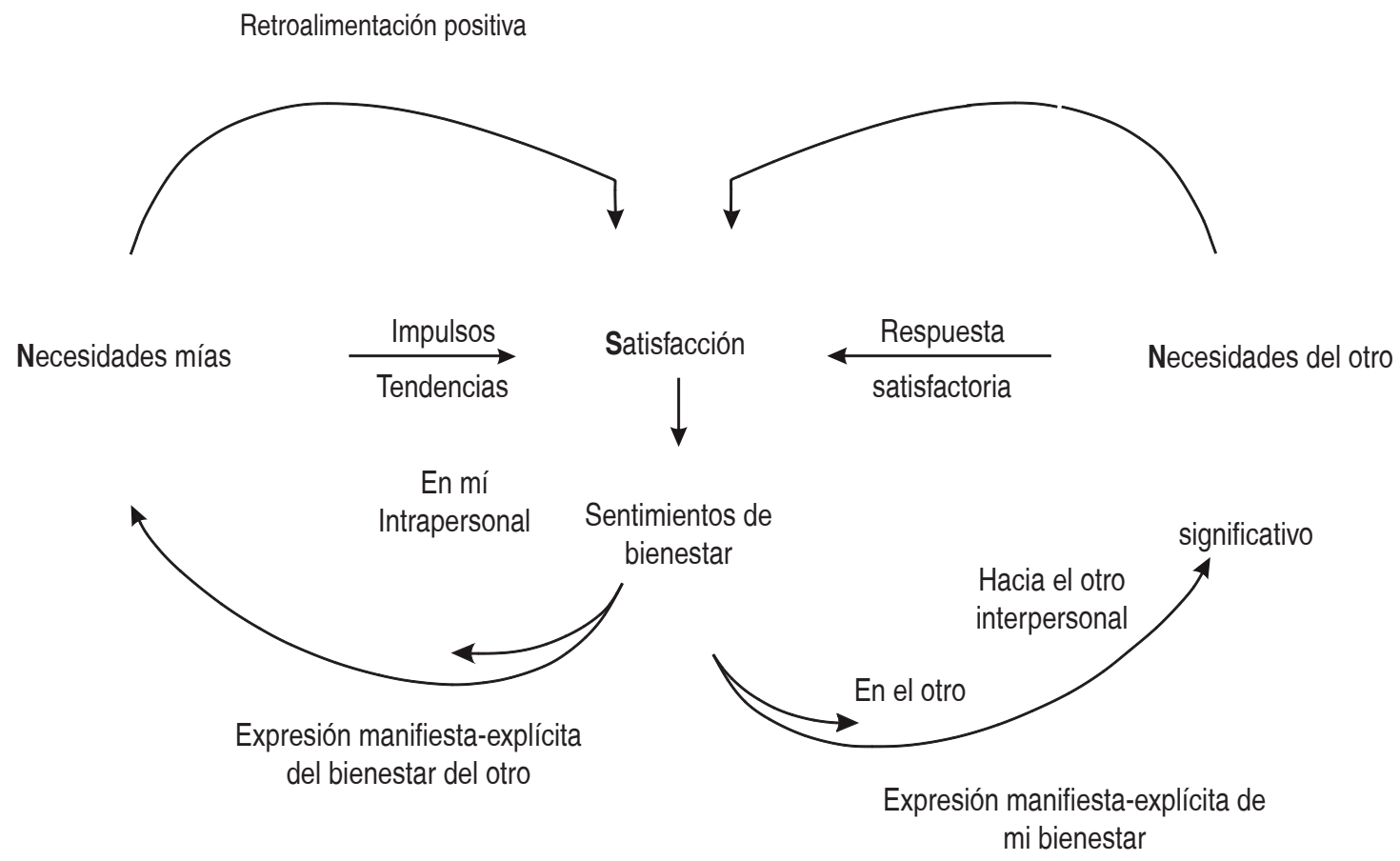

Figura 6: Circuito de doble giro inverso de retroalimentación positiva.

R. Macías-A.

3. No se tiene conciencia, no se expresan, inconsciente.

\section{Procesos y CIRCUITOS RECURSIVOS QUE GENERAN BIENESTAR, MALESTAR Y CONFLICTO}

En los siguientes diagramas tratamos de representar cómo de nuestras necesidades básicas se derivan impulsos y tendencias, que buscan su satisfacción. Para las más específicamente humanas, es necesario obtener una respuesta positiva del "Otro" significativo, si esto ocurre, se generan sentimientos de Bienestar, tanto intra- como interpersonales, pero como el "otro" tiene necesidades semejantes, es necesario que yo exprese en forma explícita y manifiesta mi bienestar y al hacerlo respondo a sus necesidades, esto genera bienestar en él y al expresarlo de la misma forma responde de nuevo a mis necesidades y refuerza mi bienestar y al expresarlo yo de nuevo, respondo a él y aumento su bienestar. De este modo se generaría un circuito recursivo, de doble giro, de reforzamiento positivo (Figura 6). Sería maravilloso mantenerlo así, pero al interrumpirse en uno y otro lado, ya no se mantiene el bienestar y se genera malestar, tanto intra- como interpersonal, que generalmente se manifiesta en expresiones en segunda persona, quejas o reproches, inculpatorios o francamente acusatorios, con frecuencia generalizantes y absolutos, que se viven como agresión y conflicto, se responde en la misma forma generando así un circuito de retroalimentación negativa de un solo giro, un franco círculo vicioso con mayor resistencia y más difícil, aunque no imposible de superar (Figura 7).

Se propone el camino del perdón y la reconciliación para ello.

\section{Concepto general de problema}

Desde una perspectiva relacional en salud mental.

Cuando una necesidad no ha podido o no puede ser satisfecha, hay un problema.

Cuando mis expectativas no se cumplen o no llegan a cumplirse, enfrento un problema.

En ambos casos se genera malestar intra- $y / 0$ interpersonal.

\section{Categorías de problemas (Figura 8)}

a. Problemas que se pueden evitar/prevenir. Prevención primaria. 


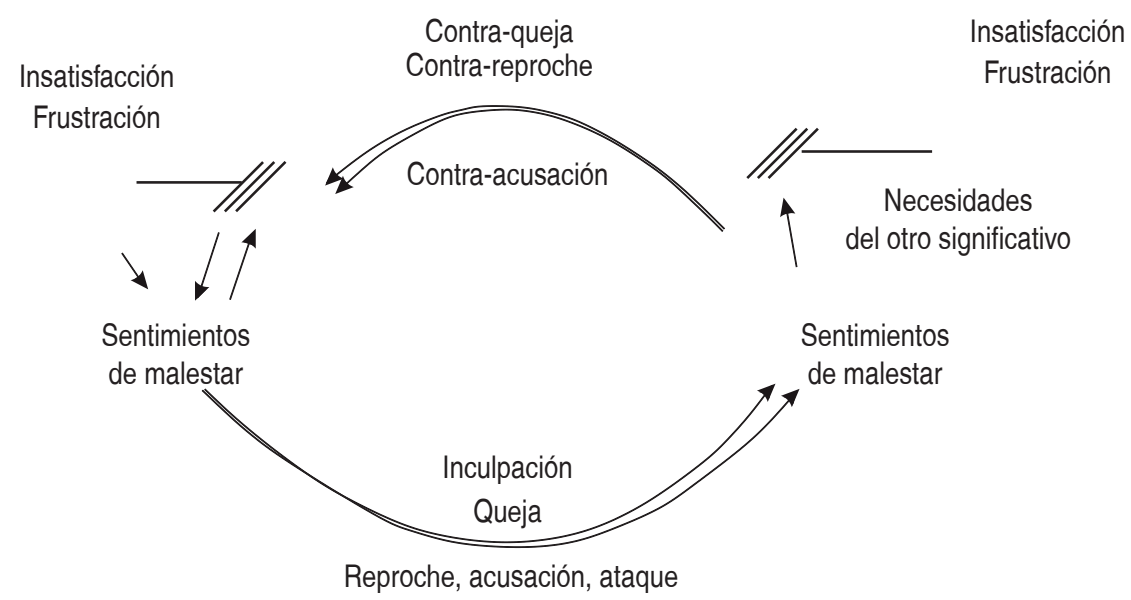

Figura 7: Circuito de un giro directo (círculo vicioso) retroalimentación negativa.

Nos atrapa en el pasado, en la culpa, en el rencor, en el no cambio (enfoque retrospectivo).

R. Macías-A.

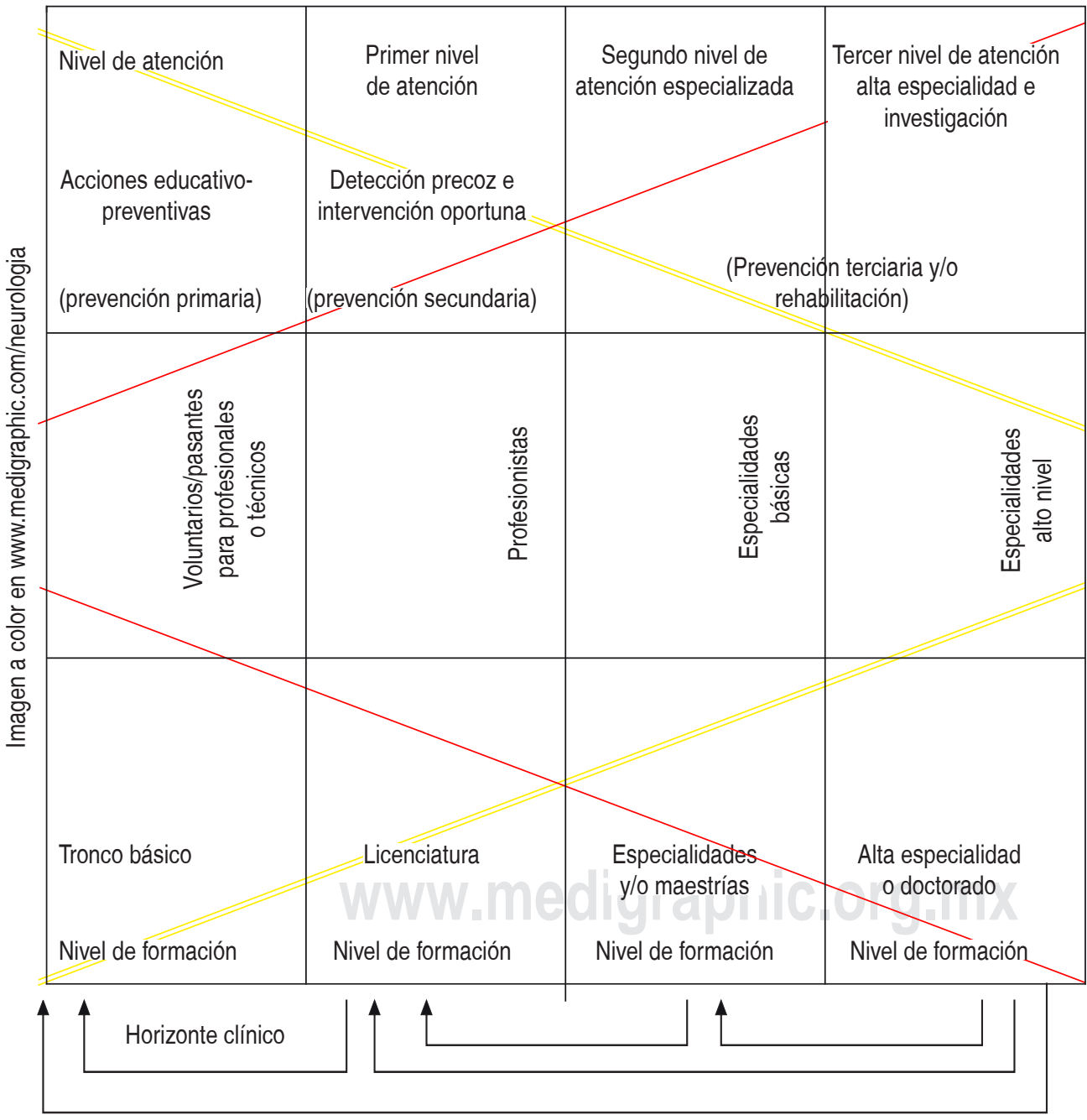

Enseñanza, supervisión, investigación, elaboración de programas.
Figura 8:

Esquema del modelo, niveles de atención.

Rojo: Complejidad de nivel de atención y formación.

Amarillo: Magnitud de la demanda y del número de prestadores de servicios.

R. Macías-A. 
b. Problemas que se pueden detectar precozmente $y$ atender/resolver temprana/oportunamente. Prevención secundaria.

c. Problemas que se pueden detectar más avanzados o tardíamente pero que aún se pueden curar o manejar adecuadamente.

d. Problemas que no se pudieron detectar ni precoz ni oportunamente, ni curar o manejar adecuadamente y hay que aprender a vivir con ellos. Prevención terciaria y/o rehabilitación.

e. Problemas mixtos con distintas combinaciones de: a, b, c y/o d.

\section{Problemáticas}

- Cambios acelerados en el mundo de hoy y la familia.

- Patrones culturales de crianza, maternaje, paternaje y salud mental.

- Derechos humanos, equidad, género, trabajo y familia.

- Violencia y abuso intrafamiliar.

- Problemas de discapacidad, enfermedad crónica y terminal.

- Dilemas éticos, educación, orientación y terapia familiar.

- Divorcio, rematrimonio y familias reconstruidas.

- Separación y migración forzada y familia.

- Discriminación, prejuicio y racismo.

- Conflicto y reconciliación, intrapersonal, interpersonal e intergrupal.

- Espiritualidad y poder curativo.

\section{NiVELES DE INTERVENCIÓN}

- Educación-promoción en salud mental (familia, escuela, comunidad, medios masivos).

- Preventivo (antes del horizonte clínico).

- Intervención en crisis (primer nivel de atención).

- Objetivos limitados.

- Modelos de terapia breve (segundo nivel de atención).

- Objetivos a mediano plazo, focalización.

- Orientados a soluciones.

- Reeducativos.

- Intervenciones sistémicas (segundo y tercer nivel de atención).

- Reconstructivos.

- Modelos interaccionales/grupales.

- Con sistemas amplios nivel comunitario.

- En contextos no clínicos.

\section{Conflicto}

\section{Concepto general}

Es un encuentro-choque de:

Fuerzas, tendencias, intenciones, conductas, emociones, sentimientos, intereses, ideas, valores, etcétera, diferentes, divergentes, contrarias, opuestas, que provoca malestar, intra y/o interpersonal, a veces muy profundo y destructivo, en diferentes niveles o ámbitos.

\section{Niveles de conflicto}

I. Intrapsíquico o intrapersonal inconsciente -consciente.

II. Interpersonal dentro de la relación de pareja o familia o fuera de ella con otro u otros significativos.

III. A nivel de grupos o sistemas diferentes, en los ámbitos social, étnico, religioso, político, ideológi$\mathrm{Co}$, generacional, regional, nacional o de bloques.

Petición

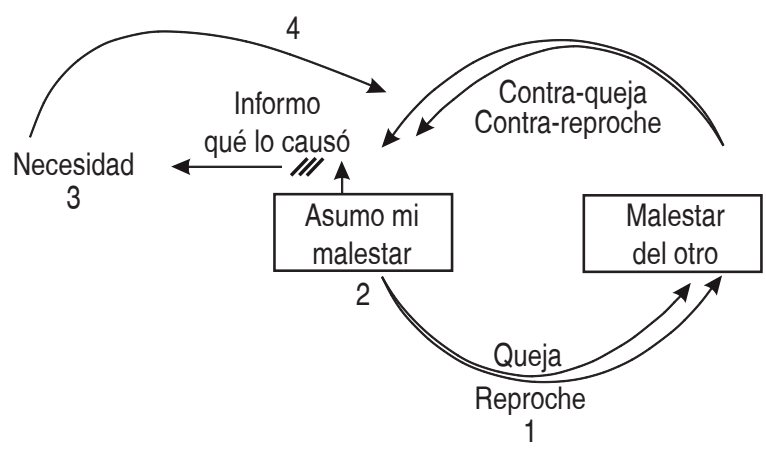

1. En vez de expresiones en segunda persona (o tercera) con depositación de la culpa en el otro, generalizantes (siempre, nunca) y juicios absolutos ("eres...").

2. Expresiones en primera persona (me siento mal, estoy enojado/a, triste), presente, describo hechos concretos del pasado reciente, informo, no juzgo.

3. Lo ligo a mi necesidad insatisfecha pasada, presente y futura (enfoque transcriptivo).

4. Petición concreta, realista, nueva posibilidad de satisfacción de bienestar, de reencuentro. Reconciliación (enfoque prospectivo al cambio).

R. Macías-A.

Figura 9: Para salir del conflicto. 


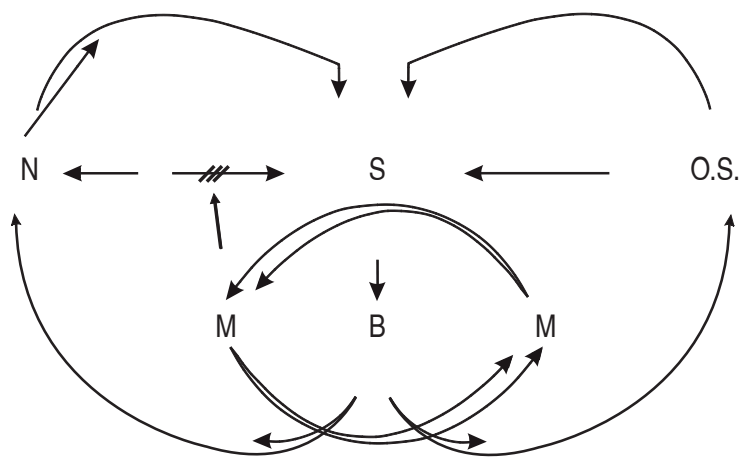

$\mathrm{N}=$ necesidad; $\mathrm{S}=$ satisfacción; $\mathrm{O} . \mathrm{S}$ = otro significativo; $\mathrm{B}=$ bienestar; $\mathrm{M}=$ malestar.

\section{R. Macías-A.}

Figura 10: Salir del círculo negativo y volver al circuito de retroalimentación positiva.

\section{Permanencia del Conflicto}

Con mucha frecuencia el conflicto se mantiene por un circuito recursivo de reforzamiento o retroalimentación negativa que nos ata al pasado y previene o evita la posibilidad del cambio y la construcción de un futuro nuevo, diferente, que generalmente en el fondo es lo que anhelamos (Figuras 9 y 10).

\section{CAMbio Y SUPERACIÓN}

Para lograr el cambio, superar el conflicto para reconciliar las fuerzas o sentimientos dentro de nosotros así como con otros y entre grupos o sistemas es imperativo liberarnos del resentimiento, del rencor, del odio, que nos esclavizan y atan al culpar, a los deseos e impulsos de venganza y de castigar, asumiendo el rol de fiscales, jurados, jueces y verdugos en uno solo, de los otros o de nosotros mismos, en un supuesto celoso afán de "justicia".

\section{UN CAMINO}

Un camino nada fácil, más bien un modo muy difícil; sin embargo, factible, y por no decir el único, de alcanzar dicho cambio, de liberarnos de las cadenas que nos atan a un pasado imposible de cambiar, a través de la culpa de mí o del otro y lograr al fin la reconciliación conmigo o con el otro, es a través del perdón, de un perdón final como renuncia al derecho a reclamar, a seguir pasando una y otra vez la factura por la misma ofensa, queja, falta, agravio o daño.

\section{LECTURAS RECOMENDADAS}

1. Fromm E. El miedo a la libertad. Buenos Aires: Paidos, 1964.

2. Fromm E. El corazón del hombre. México: FCE, 1966.

3. Epstein NB, Bishop DS. Problem-centered systems therapy of the family. En: Gurman, AS, Kniskern, DP, eds. Handbook of Family Therapy. New York: Brunner/Mazel, 1981: 444-482.

4. Macías-Avilés R. Entendiendo y tratando el corazón de la familia. México: El Saber, 2012.

5. Linton R. La historia natural de la familia. En: Fromm, E, Horkheimer, M, Parsons, T y otros: La Familia. Barcelona: Ed. Península, 1998: 5-29.

6. Ackerman NW. Diagnóstico y tratamiento de las relaciones familiares. Buenos Aires: Ed. Hormé, 1961.

7. Fromm E. Ética y Psicoanálisis. México: FCE, 1971.

8. Erikson EH. Childhood and Society. New York: Norton, 1963.

Correspondencia:

Raymundo Macías Avilés

E-mail: rcmaif@aol.com 